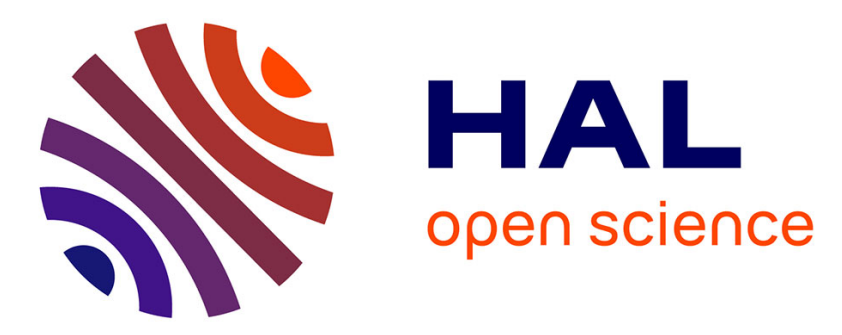

\title{
Strain Localisation and Damage Measurement by Full 3D Digital Image Correlation: Application to 15-5PH Stainless Steel
}

\author{
Tong Wu, Michel Coret, Alain Combescure
}

\section{- To cite this version:}

Tong Wu, Michel Coret, Alain Combescure. Strain Localisation and Damage Measurement by Full 3D Digital Image Correlation: Application to 15-5PH Stainless Steel. Strain, 2011, 47 (1), pp.49-61. 10.1111/j.1475-1305.2008.00600.x . hal-01007323

\section{HAL Id: hal-01007323 \\ https://hal.science/hal-01007323}

Submitted on 12 Jun 2017

HAL is a multi-disciplinary open access archive for the deposit and dissemination of scientific research documents, whether they are published or not. The documents may come from teaching and research institutions in France or abroad, or from public or private research centers.
L'archive ouverte pluridisciplinaire HAL, est destinée au dépôt et à la diffusion de documents scientifiques de niveau recherche, publiés ou non, émanant des établissements d'enseignement et de recherche français ou étrangers, des laboratoires publics ou privés. 


\title{
Strain Localisation and Damage Measurement by Full 3D Digital Image Correlation: Application to I5-5PH Stainless Steel
}

\author{
T. Wu, M. Coret and A. Combescure \\ Universite de Lyon, INSA-Lyon, LaMCoS, CNRS UMR5259, F6962I Villeurbanne, France
}

\begin{abstract}
The objective of this paper is to propose a method for measuring damage in ductile materials, from its inception to rupture. In the first stage of damage, which occurs before localisation, the usual method for determining damage through the measurement of stiffness variation is used. A damageable elastic-plastic model of the modified Lemaitre/Chaboche type is identified from these tests. An original method is proposed for measuring damage following the initiation of strain localisation. This method is based on a full 3D image correlation analysis using four cameras. The principle of the method consists in identifying the damage through tensile experiments on thin, flatnotched specimens subjected to tensile loading. Speckles are applied on both faces of each specimen in order to follow the strain fields on the two faces at the same time. These two strain fields are digitised simultaneously by two synchronised sets of two digital cameras. This paper shows how this method enables one to identify strain localisation and deduce the evolution of damage directly. Here, the method is developed for 15-5 PH stainless steel.
\end{abstract}

KEY WORDS: 3D digital image correlation, continuum damage mechanics, ductile fracture, strain localisation

\section{Introduction}

The fracture of materials is a complex process in which the mechanical properties deteriorate through damage and cracking because of volume or surface discontinuities. In the case of ductile materials, the initiation phase of the damage is called diffuse necking and results essentially in a loss of stiffness. This is followed by an accumulation of damage leading invariably to strain localisation, also called localised necking, and ends with rupture of the material. The type of fracture observed is analysed in the second part of the paper, in which fracture is observed on the microscale.

Here, the damage models used for analysing the diffuse damage phase are defined within the framework of the thermodynamics of irreversible processes [1]. Some models use cavities or porous solid plasticity to represent damage [2,3]. These theories, which assume damage to be isotropic, use a single scalar variable defined as the volume fraction of cavities in a representative volume element. Other models are based on continuum damage mechanics (CDM) [4, 5]. In CDM, damage is associated with the critical strain energy density in the constitutive equations governing the irreversible processes in the material. In the case of ductile materials, these two families of models represent, through different approaches, a single phenomenon: the growth of microvoids [6].

In this paper, we attempt to show how damage can be measured from its inception to fracture, which is still a difficult problem. The section succeeding the subsequent one focuses on the measurement of damage during diffuse necking. A usual method in which damage is measured directly through the stiffness variation of metallic specimens is reviewed. These measurements enable one to identify a damage model of the Lemaitre type [6] which has been modified by the authors $[7,8]$.

The succeeding section proposes a new methodology for measuring damage during localised necking. This direct method circumvents the delicate problem of inverse identification one usually has to deal with [3]. In order to do that, one uses flat-notched specimens (FNS), the strains of which on both faces are measured by 3D stereocorrelation. Thus, one obtains the local surface strain field and the thickness field throughout the test, which enables one to deduce damage as a local volume increase. 
Table I: Chemical composition of $15-5 \mathrm{PH}$ stainless steel (\% mass)

\begin{tabular}{lllllllllllll}
\hline $\mathrm{C}$ & $\mathrm{Si}$ & $\mathrm{Mn}$ & $\mathrm{P}$ & $\mathrm{S}$ & $\mathrm{Cr}$ & $\mathrm{Mo}$ & $\mathrm{Ni}$ & $\mathrm{Cu}$ & $\mathrm{Al}$ & $\mathrm{N}$ & $\mathrm{NB}$ & $\mathrm{Fe}$ \\
\hline 0.030 & 0.40 & 0.66 & 0.020 & 0.001 & 15.44 & 0.05 & 4.50 & 3.16 & 0.013 & 0.0247 & 0.292 & Balance \\
\hline
\end{tabular}

This research study was applied to a $15-5 \mathrm{PH}$ stainless steel alloy (AMS5659), a high-strength member of the martensitic precipitation-hardened stainless steel family which is commonly used for aerospace components such as valves, shafts, fasteners, fittings or gears. The specific $15-5 \mathrm{PH}$ we used in our experiment is H1025 (Condition H1025), i.e. a precipitation-hardened steel which has been heat solution-treated at $1025 \pm 15 \mathrm{~F}$ for $4 \mathrm{~h}$, then aircooled. The chemical composition of $15-5 \mathrm{PH} \mathrm{H} 1025$ is shown in Table 1. On the microscopic scale, this steel is martensitic at low temperatures and austenitic at high temperatures.

\section{Evaluation of the Microscopic Damage}

Microscopic observations were performed in order to study the damage mechanism on the microscale and to help choose an appropriate damage model.
Figure 1 shows $15-5 \mathrm{PH}$ microstructures under various strain levels. In the absence of strain (Figure 1A), the material's microstructure is quite smooth, except for a few microvoids and microcracks that can be regarded as initial damage. As the applied strain increases, the grains get twisted and the surface of the microvoids gets slightly distorted (Figure 1B). Under even higher strains, dimples can be observed on the surface (Figure 1C) and dimpled ductile damage occurs. Figure $1 \mathrm{D}$ is a magnified view of Figure 1C.

These experimental observations of the microstructure provide basic information on the damage mechanism in traction. A damage model of the Kachanov type was considered to be reasonable for this type of situation. The microvoid distribution and evolution observed indicate that ductile damage is induced by plastic strain, which is the basic assumption of Lemaitre and Chaboche's ductile model [6].
(A)

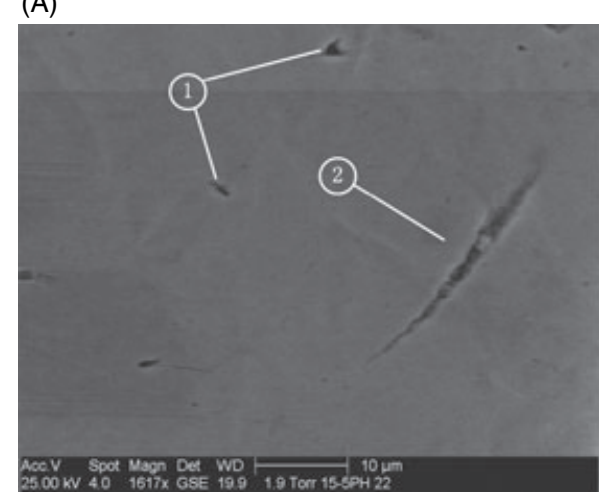

(C)

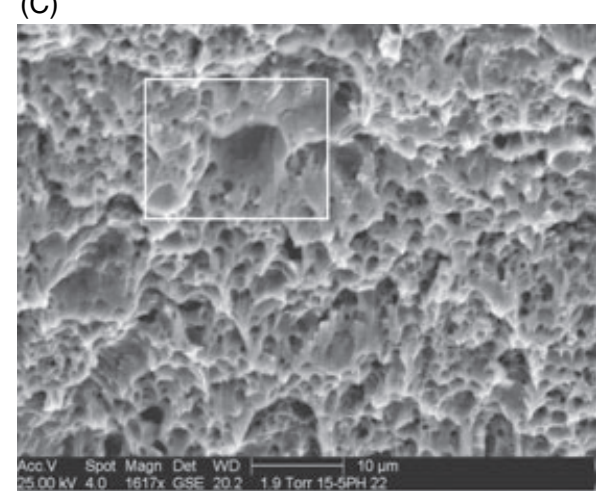

(B)

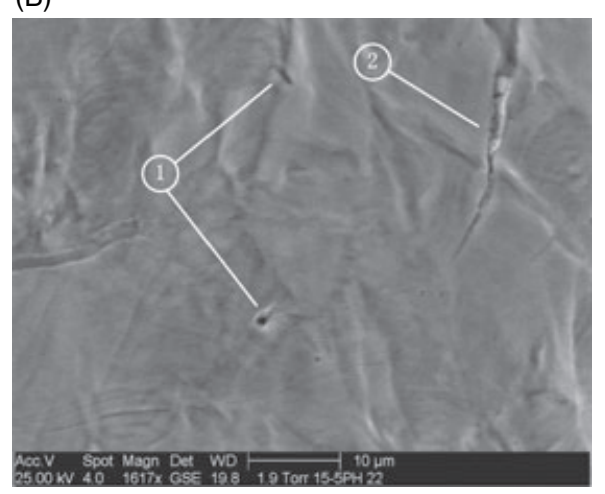

(D)

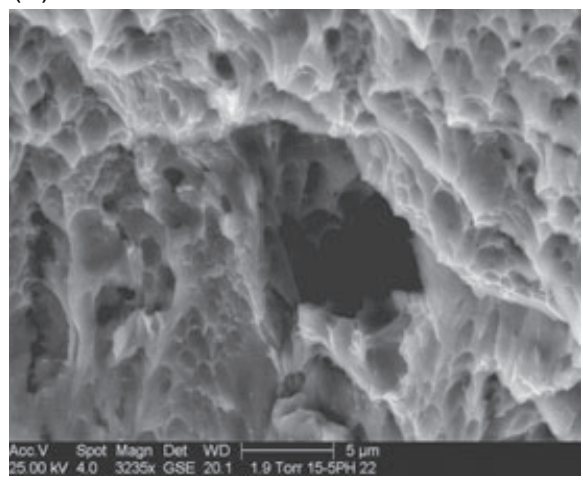

Figure I: Scanning electron microscope images of $15-5 \mathrm{PH}$ specimens under various strain loading cases at room temperature. (A) Plastic strain $p=0 \%, 1600 \times$; (B) plastic strain $p=10 \%, 1600 \times$; (C) plastic strain $p=20 \%, 1600 \times$; (D) plastic strain $p=20 \%$, 3200× 


\section{Identification of Macroscopic Damage on Standard Round Bar Specimens}

\section{Theoretical model}

The damage variable, as introduced by Kachanov [4], is defined based on the irreversible processes leading to nucleation and to the growth of microvoids and microcracks in the entire volume of the specimen. All types of voids and cracks (both intergranular and transgranular) which lead to deterioration of the integrity of the material are accounted for. For homogeneous and isotropic damage, the damage variable can be defined in the representative volume element (RVE):
$D(0)=D_{0}$

The damage exponent $\alpha$ is an additional material parameter. Finally, our model has five material parameters $\left(p_{\mathrm{D}}, p_{\mathrm{R}}, D_{0}, D_{c}, \alpha\right)$, whereas Lemaitre and Chaboche's basic ductile model has only three material damage parameters $\left(p_{\mathrm{D}}, p_{\mathrm{R}}, D_{\mathrm{c}}\right)$. It is obvious that our model reduces to Lemaitre and Chaboche's model if $D_{0}=0$ and $\alpha=1$. The identification of this model consists in setting the values of the five coefficients by means of tensile tests.

Let us observe that in most structures the applied stress is not uniaxial. Following Lemaitre and Chaboche's method of obtaining 3D models $[5,6]$, the 3D damage model is described in rate form by the following equation:

$\dot{D}= \begin{cases}\frac{D_{\mathrm{c}}-D_{0}}{\left(p_{\mathrm{R}}-p_{\mathrm{D}}\right)^{\alpha}} \alpha \Omega\left(\frac{\sigma_{\mathrm{H}}}{\sigma_{\mathrm{eq}}}\right)\left(p \Omega\left(\frac{\sigma_{\mathrm{H}}}{\sigma_{\mathrm{eq}}}\right)-p_{\mathrm{D}}\right)^{\alpha-1} \dot{p} & \text { if } \sigma_{\mathrm{H}} \geq 0 \text { and } p \Omega\left(\frac{\sigma_{\mathrm{H}}}{\sigma_{\text {eq }}}\right)>p_{\mathrm{D}} \\ 0 & \text { if } \sigma_{\mathrm{H}}<0 \text { or } p \Omega\left(\frac{\sigma_{\mathrm{H}}}{\sigma_{\mathrm{eq}}}\right) \leqslant p_{\mathrm{D}}\end{cases}$

$D=\frac{S_{\mathrm{D}}}{S}$

where $S$ is the area of the intersection of the plane being considered with the RVE and $S_{\mathrm{D}}$ is the effective area of the intersections of all microcracks and microvoids in $S$.

Following the thermodynamic approach of Lemaitre and Chaboche [5], the damage rate variable is related to the equivalent plastic strain rate. In the case of uniaxial ductile plastic damage strain, the model is the following:

$\dot{D}= \begin{cases}\frac{D_{\mathrm{c}}}{p_{\mathrm{R}}-p_{\mathrm{D}}} \dot{p} & \text { if } p>p_{\mathrm{D}} \text { and } \sigma>0 \\ 0 & \text { otherwise }\end{cases}$

where $D_{\mathrm{c}}$ represents the critical damage, $p$ the accumulated equivalent plastic strain and $p_{\mathrm{R}}$ its value at rupture. $p_{\mathrm{D}}$ is the threshold-equivalent accumulated plastic strain and $\sigma$ is the applied stress. The dot symbol denotes the time derivative.

The experimental damage results of $15-5 \mathrm{PH}$ show that in a uniaxial tensile test the damage is a nonlinear function of the plastic strain. In order to match the experimental data, we propose to introduce an initial damage $D_{0}$ and a power law characterised by an exponent $\alpha$. For uniaxial loading, we get:

$$
\dot{D}= \begin{cases}\left(D_{\mathrm{c}}-D_{0}\right) \alpha \dot{p}\left(\frac{p}{p_{\mathrm{R}}-p_{\mathrm{D}}}\right)^{\alpha-1} & \text { if } p>p_{\mathrm{D}} \text { and } \sigma>0 \\ 0 & \text { otherwise }\end{cases}
$$

where

$$
\Omega\left(\frac{\sigma_{\mathrm{H}}}{\sigma_{\mathrm{eq}}}\right)=\frac{2}{3}(1+v)+3(1-2 v)\left(\frac{\sigma_{\mathrm{H}}}{\sigma_{\mathrm{eq}}}\right)^{2} \text { and } D_{0}=D(0)
$$

In Equation (4), $\sigma_{\mathrm{H}}$ is the hydrostatic stress while $\sigma_{\mathrm{eq}}$ is von Mises' equivalent stress and $v$ is the Poisson's ratio. Here, let us observe that this 3D damage model differs from the 1D model by the presence of the triaxiality stress ratio, which may modify the damage rate drastically (e.g. $\dot{D}=0$ if $\sigma_{\mathrm{H}}<0$ ). The elastic-plastic strain rates are coupled with the damage rate. The full description of the model can be found in Ref. [7].

\section{Tests}

The tests were performed at room temperature on a servo-electrohydraulic tension-compression machine with a maximum capacity of $250 \mathrm{kN}$. The specific strain measurement system used, dedicated to hightemperature strain measurements, can be seen in Figure 2. An extensometer with $1 \mu \mathrm{m}$ precision (consisting of two sticks, connecting components, a fixing component and conducting wires) was used to measure the axial strain. The two sticks were placed parallel to each other at a gauged distance of $15 \mathrm{~mm}$ to serve as the basis for the strain measurements. The high-temperature results are not presented in this paper. 


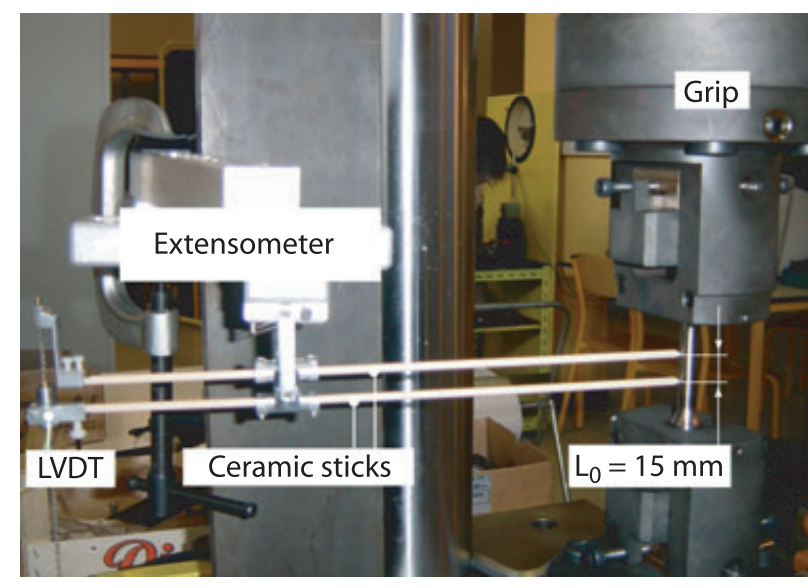

Figure 2: Experimental setup for the round bar tests

The round bar (RB) cyclic load-unload tests not only provided stress-strain curves and mechanical properties such as Young's modulus, yield strain, yield stress and hardening behaviour, but were also used to identify the damage variable $D$. The round specimens were loaded progressively and unloaded at different stages of the loading to evaluate the evolution of the damage with plastic strain. The geometry of the RB specimens is shown in Figure 3.

\section{Measurements}

The global load and displacement were measured by the loading system of the tensile test machine, consisting of a load cell and a linear variable displacement transducers (LVDT) sensor, and recorded in the controller system (PC component). The axial strain

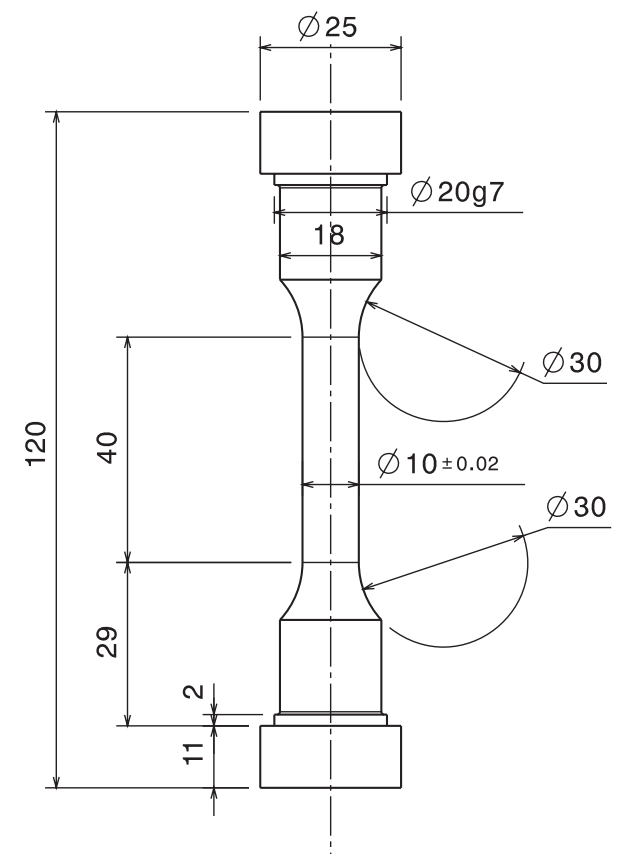

Figure 3: Geometry and dimensions of the RB specimens (units: $\mathrm{mm}$ ) was measured by the previously described extensometer (see section 'Tests').

Damage cannot be easily measured directly. Its quantitative evaluation is linked to the definition of the variable chosen to represent the phenomenon. The macroscopic global damage can be measured through the modification of the Young's modulus. This indirect measurement approach gives a first insight into the evolution of damage (see Figure 4).

Let us review the concept of effective stress [4]:

$\underline{\underline{\underline{\sigma}}}=\frac{\underline{\underline{\underline{\sigma}}}}{1-D}=\underline{\underline{\underline{\underline{H}}}} \underline{\underline{\underline{\varepsilon_{\mathrm{e}}}}}$

where $H$ is Hooke's matrix, $\tilde{\sigma}$ the effective stress tensor $\overline{\overline{\bar{a}}} d \varepsilon_{\mathrm{e}}$ the elastic strain. In the uniaxial case, this equation becomes:

$\tilde{\sigma}=\frac{\sigma}{1-D}=E \varepsilon_{\mathrm{e}}$

$E(1-D)=\tilde{E}$ can be interpreted as the elastic modulus of the damaged material. The damage variable $D$ can be written as:

$D=\frac{1-\tilde{E}}{E}$

where $E$ and $\tilde{E}$ are the Young's moduli of the undamaged material and damaged material, respectively.

Despite its apparent simplicity, such a measurement is rather tricky because of the following reasons:

1 The measurement of the modulus of elasticity requires precise measurements of very small strains.

2 The damage is usually localised and, thus, the extensometry technique can be applied only to a limited zone.

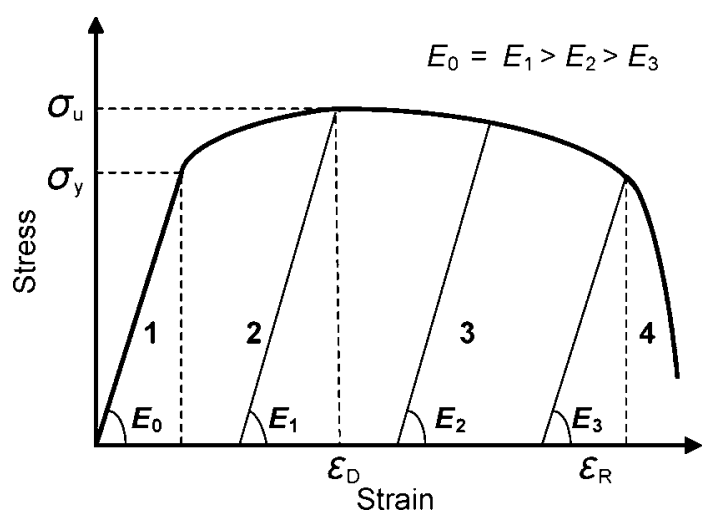

Figure 4: Stress/strain diagram and evolution of the Young's modulus (zone 1: elastic stage; zone 2: elastic-plastic stage; zone 3: elastic-plastic stage in which the damage occurs; zone 4 : initiation and propagation of cracks leading to final rupture) 
3 In the stress-strain graph, it is difficult to identify the 'best straight line' which represents elastic loading or unloading.

The unloading ramp is used to measure the Young's modulus. In evaluating the modulus of elasticity during elastic unloading, one must stay away from highly nonlinear zones. This is achieved by carrying out the evaluation in a stress range defined as follows:

$\sigma_{\min }<\sigma<\sigma_{\max }$

where $\sigma_{\min }$ and $\sigma_{\max }$ are the lower and upper stress limits of the linear part, respectively.

In the stress-strain region defined above, there are two errors: a strain error and a stress error. Therefore, the true measured value lies somewhere within a small rectangle. The strain error is $5 \times 10^{-5}$ and the stress error was $0.5 \mathrm{MPa}$ in our tests. Of course, the previous method is not valid in the case of localisation because the damage cannot be considered to be uniform throughout the specimen.

\section{Remark}

As usual, the measured strain (total strain) is assumed to be the sum of an elastic strain $\underline{\underline{\underline{\varepsilon_{\mathrm{e}}}}}$ and an irreversible plastic strain $\underline{\underline{\varepsilon_{\mathrm{p}}}}$.

\section{Experimental results of the RB tests}

The experimental results obtained from the RB tensile tests are the following: the initial Young's modulus was $199 \mathrm{GPa}$, the yield stress $\sigma_{\mathrm{y}}=861 \pm$ $0.5 \mathrm{MPa}$, the $0.2 \%$ yield stress $\sigma_{0.2}=1028 \pm 0.5 \mathrm{MPa}$ and the ultimate stress $\sigma_{\mathrm{u}} 1264 \pm 0.5 \mathrm{MPa}$. The stress-strain curve with unloading is shown in Figure 5A.

Figure $5 \mathrm{~A}$ shows that the hardening stage is followed by a softening stage before final rupture. The material breaks sharply. The unloading enables the evaluation of the variation of the Young's modulus, which leads to the determination of the damage variable. Figure $5 \mathrm{~B}$ shows a zoom on the sixth unloading. The Young's modulus was determined by linear regression from the $\sigma_{\min }$ and $\sigma_{\max }$ stresses during loading and unloading. As unloading is slightly more linear than loading, the value obtained during unloading, i.e. $173 \mathrm{GPa}$, was retained.

\section{Identification of the damage model from the RB experiments}

The identification of the damage model requires the determination of several parameters. These constants and their meaning are presented below:

$D_{0}$ : initial damage. The initial damage is difficult to determine because it is related strictly to the distribution of inclusions in the virgin material's microstructure. An investigation by scanning electron microscopy can provide an estimate of the initial particle distribution and of the amount of damage in the strained area. As usual, this parameter can be assumed to be zero for a virgin material or at the beginning of the damage calculation. For example, the measurement of the damaged area in Figure $1 \mathrm{~A}$ gives the approximate value of the initial damage $D_{0} \approx 0.001$.

$p_{\mathrm{D}}$ : threshold plastic strain for damage. Plastic damage begins only past a specific strain level. Below this level, the material's microstructure behaves like a continuum. Microvoids can nucleate either by debonding of the particles involved from the ductile matrix or by particle breakage.

$p_{\mathrm{R}}, D_{\mathrm{c}}$ : failure plastic strain and critical damage. When this ultimate strain is reached, failure occurs. $p_{\mathrm{R}}$ is related directly to the amount of damage which reduces the load-carrying capacity of the effective
(A)

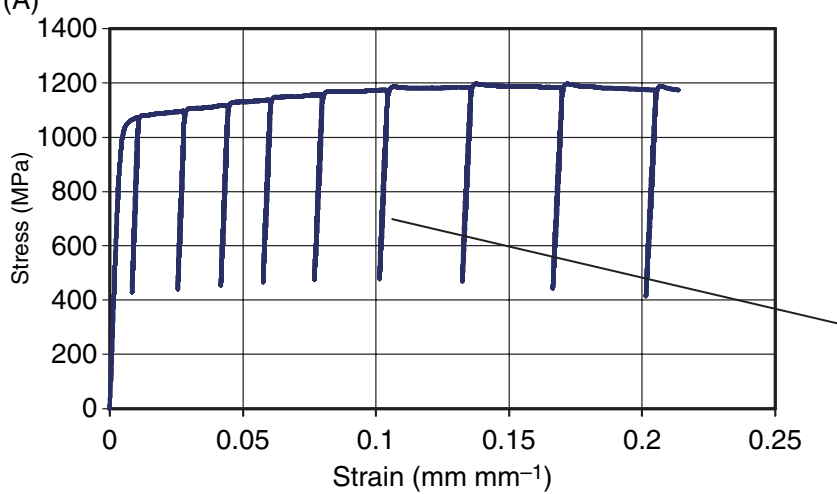

(B)

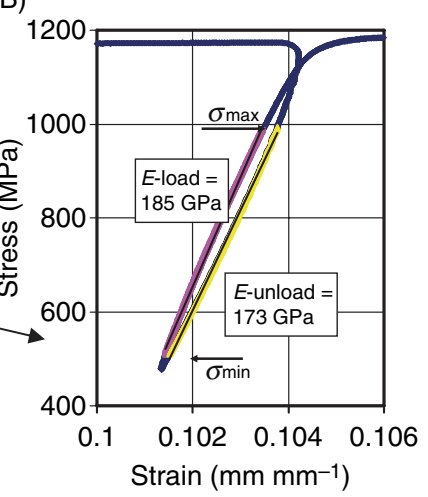

Figure 5: (A) Stress-strain curves of the cyclic load-unload tests at room temperature; (B) zoom on the sixth unloading step 
resisting section critically. In theory, when failure occurs, the critical damage variable $D_{\text {c }}$ should be equal to 1 . In fact, in almost all experimental observations on metals, failure occurs before $D=1$. $\alpha$ : damage exponent. This constant carries information which influences the type of damage evolution and can be determined from tensile tests. Its value sets the degree of nonlinearity of the damage evolution law. For any given $p_{\mathrm{D}}, p_{\mathrm{R}}, D_{0}$ and $D_{\mathrm{c}}$, there is a discriminating value of $\alpha$ which determines the convexity of the damage evolution as a function of plastic strain.

If $D_{0}$ is assumed to be zero, the time integration of Equation (6) gives:

$D=D_{\mathrm{c}}\left\langle\frac{p-p_{\mathrm{D}}}{p_{\mathrm{R}}-p_{\mathrm{D}}}\right\rangle^{\alpha}$

The exponent $\alpha$ is chosen as the slope of the bestfitting line of the experimental damage measurements given by:

$\ln (D)=\ln \left(D_{\mathrm{c}}\right)+\alpha \cdot \ln \left\langle\frac{p-p_{\mathrm{D}}}{p_{\mathrm{R}}-p_{\mathrm{D}}}\right\rangle$

Hence, one has:

$\ln (D)=\alpha \cdot \ln \left(p-p_{\mathrm{D}}\right)-C$

with:

$C=\alpha \cdot \ln \left(p_{\mathrm{R}}-p_{\mathrm{D}}\right)-\ln \left(D_{\mathrm{C}}\right)$

where the constant $C$ is the intersection of the fitting line with the ordinate axis. Usually, the failure strain and the critical damage can be measured, but the threshold strain is difficult to determine because of the experimental scatter.

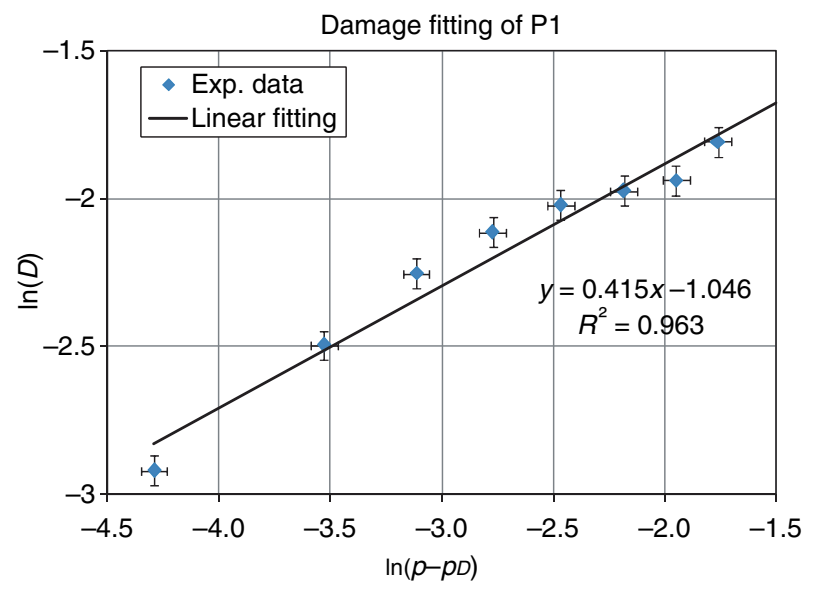

Figure 6: Fitting of the damage parameters at room temperature
Once the slope has been found, one can get a reasonable estimate of $p_{\mathrm{D}}$ :

$p_{\mathrm{D}}=p_{\mathrm{R}}-\exp \left(\frac{C+\ln \left(D_{\mathrm{c}}\right)}{\alpha}\right)$

Figure 6 shows the fitting of parameters $C$ and $\alpha$ for a martensitic state at $20{ }^{\circ} \mathrm{C}$. The error bars were calculated from the stress and strain measurements (see section 'Measurements') The identified parameters are $p_{\mathrm{D}}=0.010, p_{\mathrm{R}}=0.21, D_{\mathrm{c}}=0.18, C=1.02$ and $\alpha=0.42$. The value of the critical damage is small, which is quite often the case for metals (see Refs [5, 6]). This could be due to the type of identification chosen, which relies on a displacement measurement based on a 15-mm distance between the sticks to evaluate the strains.

\section{D Digital Image Correlation for the Identification of Localisation and Damage on FNS}

We designed tests on FNS with three different notch radii: $R=1.0 \mathrm{~mm}$ (case $\mathrm{A}$ ), $R=2.5 \mathrm{~mm}$ (case $\mathrm{B}$ ) and $R=4.0 \mathrm{~mm}$ (case $\mathrm{C}$ ). The strain and stress gradients vary with the geometry of the notch. The geometry of the specimens used in the test is shown in Figure 7.

Two synchronised sets of two charge-coupled device (CCD) cameras (Figure 8) were used to record digital images on both faces of the specimen. 3D digital image correlation (3D-DIC) was used to process the digital images and analyse the strain localisations. The use of both CCD cameras and image correlation made it possible to monitor strain localisation and observe the evolution of ductile damage.

\section{Displacement field measurement using 3D-DIC}

Digital image correlation has become a common surface displacement measurement technique. The idea behind this method is that the displacements of the material being tested can be obtained by tracking the deformations of a random speckle pattern applied to the component's surface through digital images acquired during the loading. Full-field displacement data are obtained, from which strains can be deduced using standard finite-element shape functions. The strain tensor used in the rest of the paper is the infinitesimal strain tensor, even when the strains are not very small.

Correlation techniques have been used on digital images by many researchers in order to measure in-plane displacement components on plane speci- 


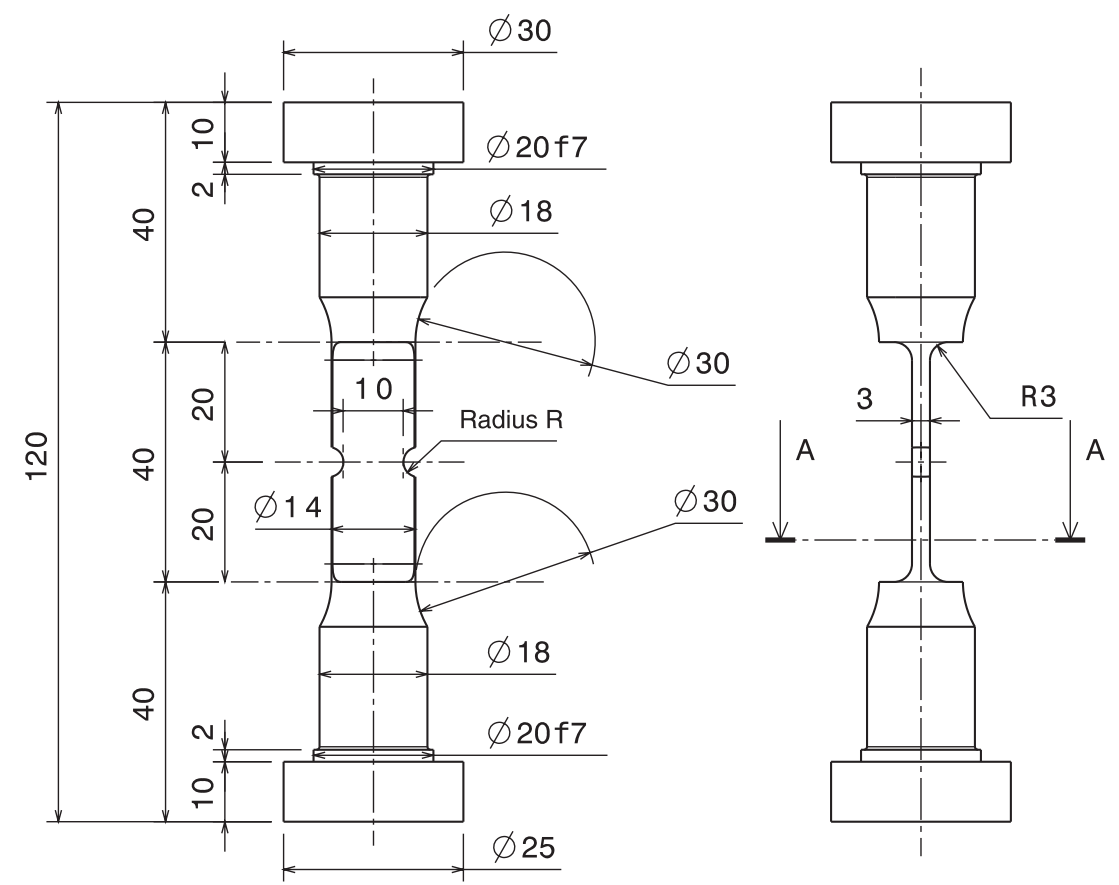

Figure 7: Geometry and dimensions of the FNS (units: $\mathrm{mm}$ )

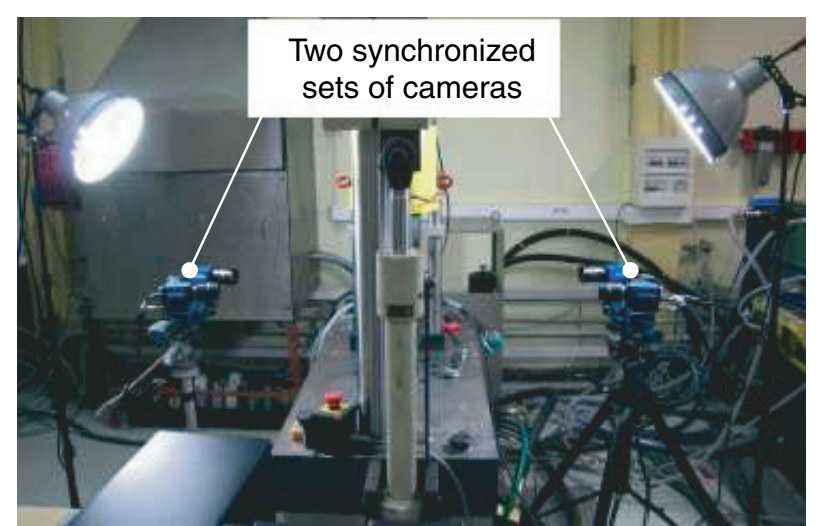

Figure 8: Flat-notch experimental setup

mens [9-12]. Helm et al. extended the DIC method by using a pair of cameras, thus enabling the measurement of 3D shapes as well as that of the 3D displacements [13]. The 3D technique requires the use of at least two synchronised cameras acquiring images of the loaded specimen from different viewing angles. By determining corresponding image locations across views from the different cameras and tracking the movement throughout the loading cycle, the shape and displacements can be reconstructed after a simple camera calibration.

In order to obtain good digital images of the specimen during tensile tests, special attention should be paid to the illumination, the speckle pattern, the choice of spray paint, the calibration of the two cameras and the paint movement with the sur- face. Reflections and shadows should absolutely be avoided. Small speckles lead to high spatial resolution. In our test, black and white spray paints were used in order to achieve a proper speckle pattern and to control the contrast gradient of the image.

Pictures were taken by four CCD cameras with a resolution of $2048 \times 2048$ pixels and a dynamic range of 8 bits (256 levels of grey). On the pictures, the approximate size of the specimen was $200 \times 400$ pixels, which corresponds to a pixel size of about $0.05 \mathrm{~mm}$. The correlation was calculated with a subset size of 15 pixels and a shift of 5 pixels. With such a setup, the strain resolution is in the $\left[1 \times 10^{-3}\right.$ to $2 \times 10^{-3}$ ] range. As in this study we wanted to measure strains greater than $1 \%$, this resolution is acceptable.

A typical FNS with speckle pattern is shown in Figure 9. Point $\mathrm{O}$ is the centre of the plate. Special attention must be paid to the displacements and strains along lines PQ (axial symmetry) and MN (horizontal symmetry).

\section{Comparative analysis of experimental FNS results}

Strain localisation evolutions on surfaces with speckle patterns were obtained by digital image correlation of tensile FNS experiments. Three cases (cases A, B, C) with different notches were analysed in order to compare their surface strains.

First, let us analyse the in-plane strains: Figure 10A shows that the notch radius has a significant impact 


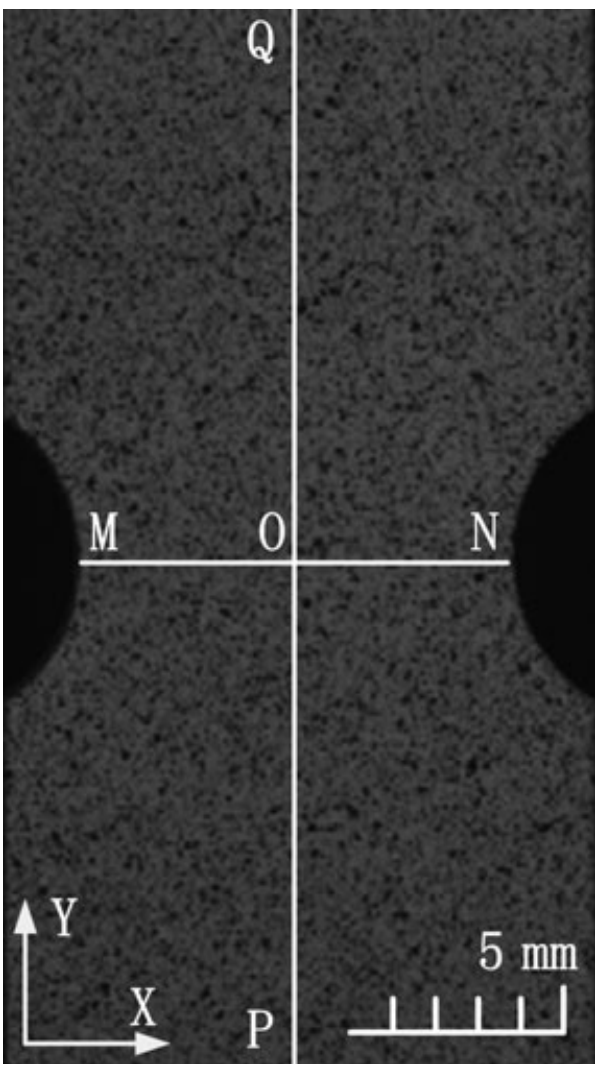

Figure 9: Flat-notched specimen with speckle pattern

on the axial strain (EPYY) along the mid-section symmetry line $\mathrm{MN}$, but has a lesser impact on the transverse strain (EPXX). Regardless of the notch radius, the highest strain is always at the edge of the notch (see Figure 10A). Figure 10B shows the strain evolution along the axial symmetry line PQ. The highest strain occurs near the mid-plane of the specimen. Figure 11 shows that the notch radius has little influence on the overall tensile curve (force versus displacement). Figure 12 shows the section reduction (the horizontal displacement difference between points $\mathrm{N}$ and $\mathrm{M}$ ) for specimens $\mathrm{A}, \mathrm{B}$ and $\mathrm{C}$ : this measurement is more discriminating.

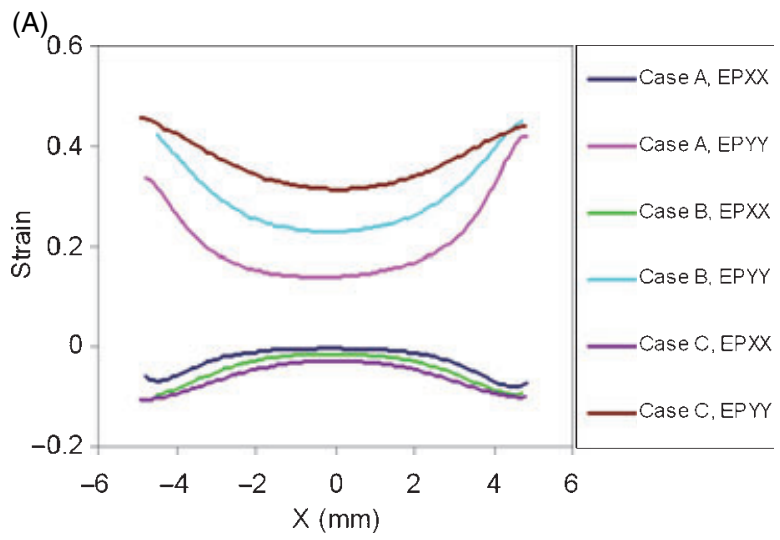

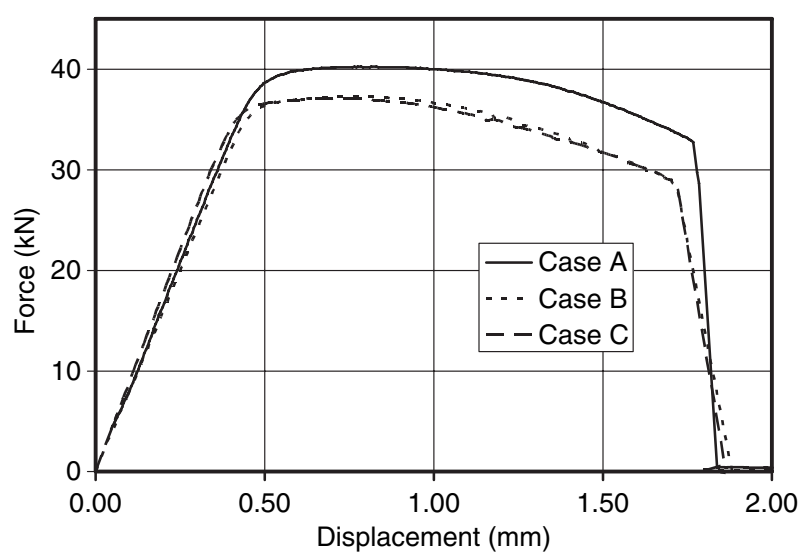

Figure II: Force versus applied displacement for FNS tensile tests of specimens $\mathrm{A}, \mathrm{B}$ and $\mathrm{C}$

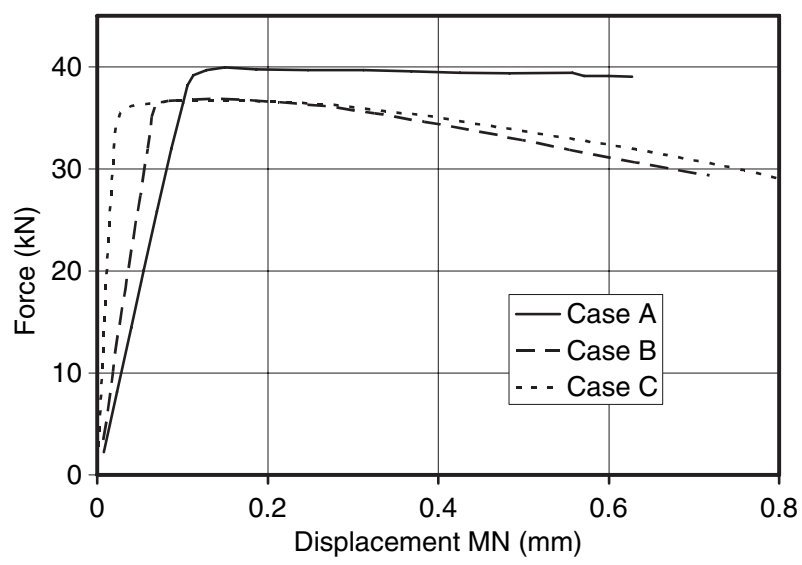

Figure 12: Force versus section reduction (between points $\mathrm{M}$ and $\mathrm{N}$, mid-section)

Figure 13 shows a field representation of the EPYY axial strains. In the three cases, the distribution is similar, with a peak strain value always occurring at the edge of the notch. Let us now analyse the outof-plane displacement distribution. Figure 14 shows the out-of-plane displacement field on one face of the specimen, which is a good indicator of thickness reduction. It is clear that this displacement is highly

(B)

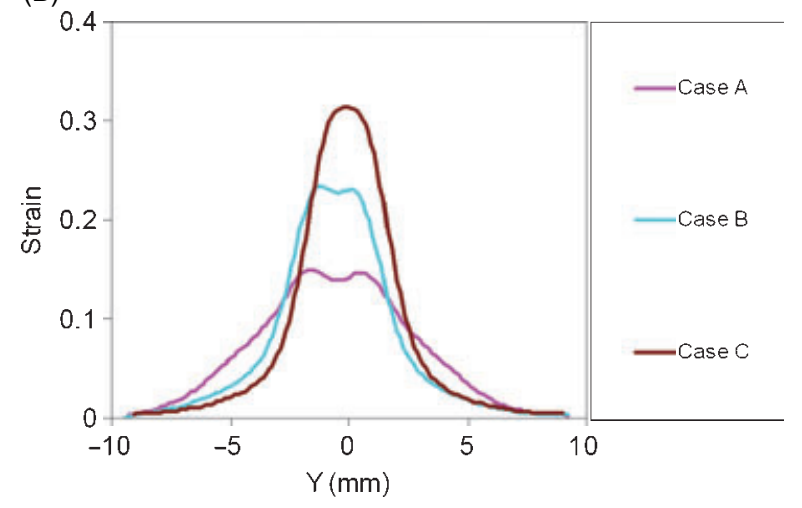

Figure 10: Comparison of the EPYY and EPXX surface strain distributions along lines MN and PQ for a $1.5 \mathrm{~mm}$ prescribed displacement. (A) EPYY and EPXX strain comparison along MN. (B) EPYY strain comparison along PQ 


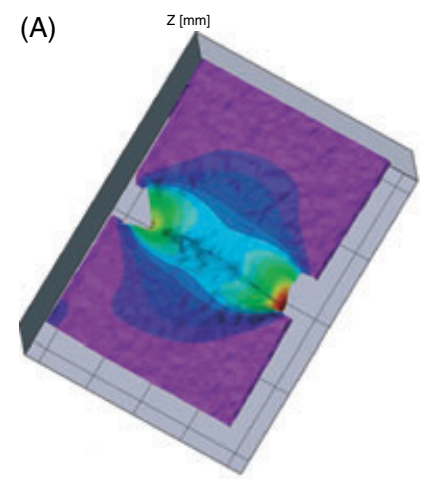

Case A

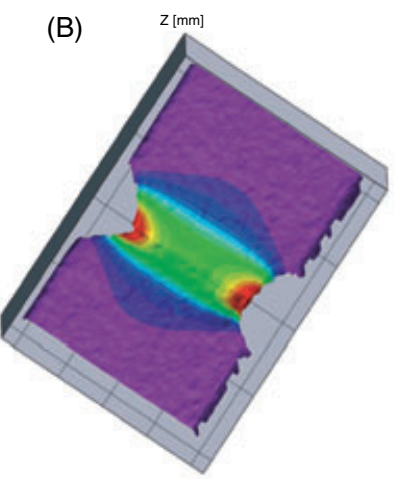

Case B

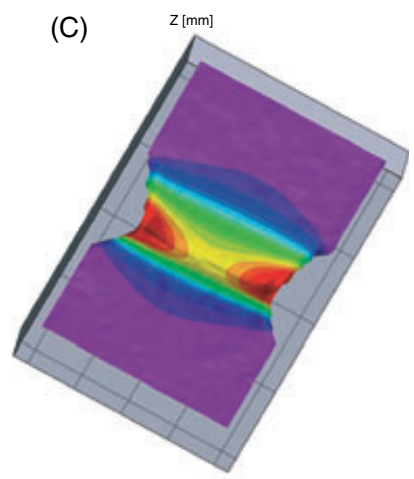

Case C 0 10 20 30 40

EPYY \%

Figure 13: DIC plot of the EPYY axial strain on notched specimens (cases A, B and C) under a $1.5 \mathrm{~mm}$ prescribed displacement $(d=1.5 \mathrm{~mm})$

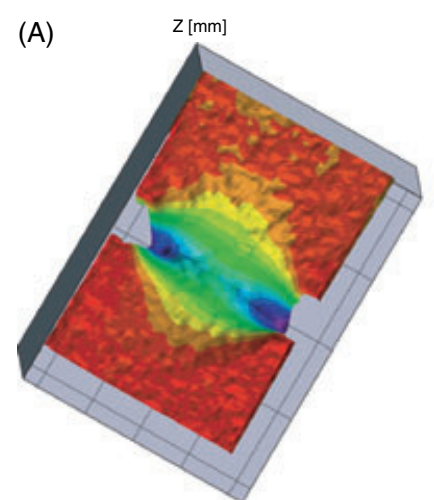

Case A

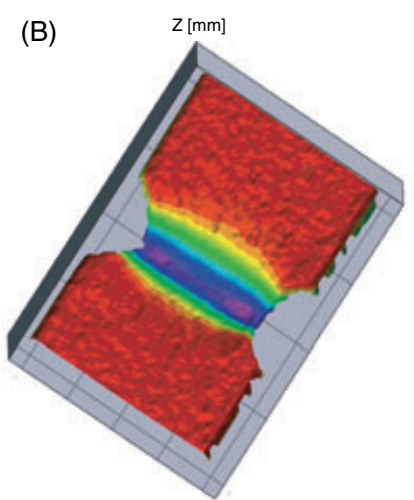

Case B

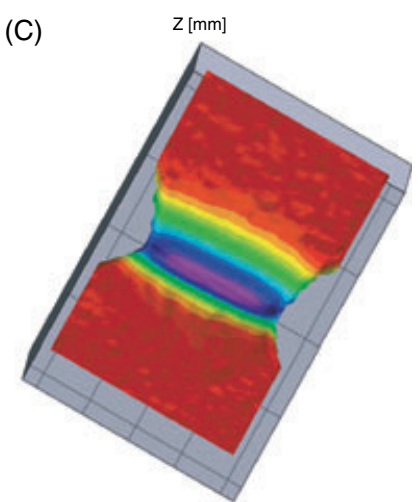

Case C

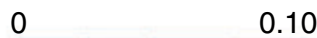

0.20

0.40

$\mathrm{UZ}[\mathrm{mm}]$

Figure 14: 3D distribution of the vertical displacement UZ of notched specimens (cases A, B and C) obtained by DIC for a $1.5 \mathrm{~mm}$ prescribed displacement $(d=1.5 \mathrm{~mm})$ uniaxial tensile test at room temperature

dependent on the notch radius. The maximum anticipated thickness reduction is near the notch edges for case $\mathrm{A}$, somewhere inside for case $\mathrm{B}$, and in the centre for case $C$.

By comparing the two out-of-plane displacement fields on the two faces of the specimen, one can calculate the thickness variation field, and the knowledge of the thickness enables one to deduce the corresponding axial strain field. The thickness reduction can be used as a rough estimate of the damage evolution, and one can consider that the damage is maximum where the section is minimum. Therefore, the comparison of Figures 13 and 14 leads to the conclusion that the highest strains and the maximum thickness reductions do not occur in the same locations: this observation is an indication that damage occurs just prior to failure. As the strains close to failure are rather high, the measured strains are mainly elastic-plastic and, therefore, the maximum strain across the thickness should be in the same location as the maximum axial strain. This is an indication that damage is developing and that the influence of the triaxiality ratio (as described by Equation (5)) is discriminating. Large-strain elasticplastic calculations of cases A, B and C (using the experimental stress-strain curve without damage) were carried out with 4500 eight-node 3D elements using the CAST3M FEM code [14]. The mesh is shown in Figure 15. The results show that the peak values of the triaxiality stress ratio are located along the edges for case A and in the centre for case $\mathrm{C}$, which is in qualitative agreement with the observations mentioned above (see Figure 14). The comparison with experimental results confirms the impression that 


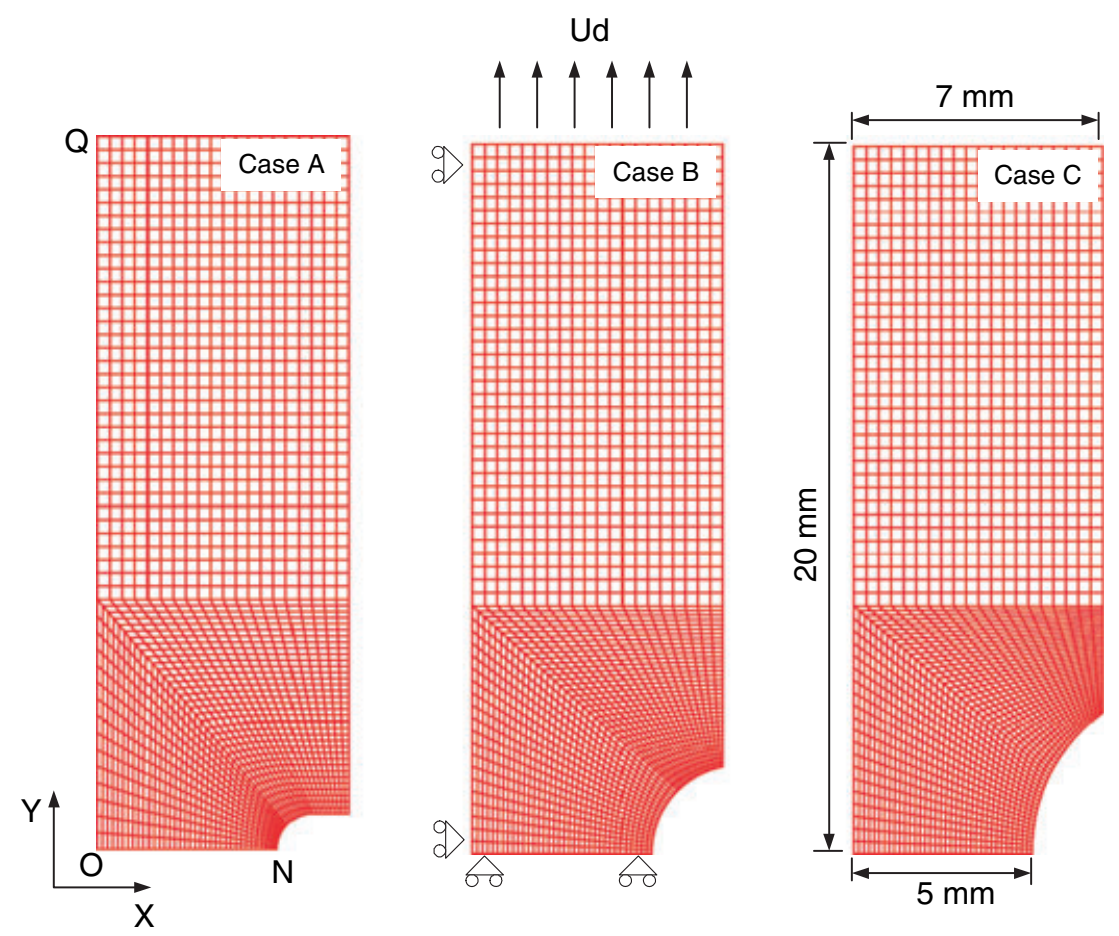

Figure 15: The meshes of the notched specimens

stress triaxiality plays an important role in the evolution of damage.

\section{Strain localisation and damage estimation}

Let us assume that the elastic strains can be neglected. This is a reasonable assumption for metals, provided the strains are greater than $1 \%$. Let us also consider a cube of initial side $l_{0}$ and initial volume $V_{0}$. After mechanical deformation, the volume of the cube is $V=\left(l_{0}+\right.$ $\left.\Delta l_{1}\right) \times\left(l_{0}+\Delta l_{2}\right) \times\left(l_{0}+\Delta l_{3}\right)$ in the space of the principal strain directions, and therefore:

$$
\left(1+\frac{\Delta l_{1}}{l_{0}}\right) \times\left(1+\frac{\Delta l_{2}}{l_{0}}\right) \times\left(1+\frac{\Delta l_{3}}{l_{0}}\right)=\frac{V}{V_{0}}=1+\frac{\Delta V}{V_{0}}
$$

$\ln \left(1+\varepsilon_{\mathrm{I}}\right)+\ln \left(1+\varepsilon_{\mathrm{II}}\right)+\ln \left(1+\varepsilon_{\mathrm{III}}\right)=\ln \left(1+\frac{\Delta V}{V_{0}}\right)$

which yields:

$\operatorname{Tr}(\mathbf{h})=\ln \left(1+\frac{\Delta V}{V_{0}}\right)$

where $\operatorname{Tr}(\mathbf{h})$ is the first invariant of the logarithmic (or Hencky's) strain tensor.

In the case of small strains, this quantity writes:

$\operatorname{Tr}(\mathbf{h}) \approx \operatorname{Tr}(\boldsymbol{\varepsilon}) \approx \frac{\Delta V}{V_{0}}$
Let us assume that the volume change is caused by the growth of voids and that damage is isotropic. Using the definition of damage of Equation (1), one gets:

$D=\frac{S_{\mathrm{D}}}{S_{0}}=\frac{\Delta S}{S_{0}}=\left(\frac{\Delta V}{V_{0}}\right)^{2 / 3}=[\operatorname{Tr}(\mathbf{h})]^{2 / 3}$

Now, one needs the full strain tensor in order to evaluate the volume variations. In standard 3D image correlation experiments using two cameras, the correlated results give access to the full $3 \mathrm{D}$ displacement field, but only to the in-plane strain because there is no information on the displacement fields on the other face. In this study, four cameras were used to observe both surfaces of the flat notched specimens and, therefore, the complete thickness information could be obtained using the difference of the $U_{Z}$ displacements between the two faces.

We make the following assumption: Let $F_{1}$ and $F_{2}$ denote the two faces of the specimen and $t$ its initial thickness. The mean strains across the thickness are given by:

$\left\langle\varepsilon_{X X}\right\rangle=\frac{1}{2}\left[\varepsilon_{X X}\left(F_{1}\right)+\varepsilon_{X X}\left(F_{2}\right)\right]$

$\left\langle\varepsilon_{y y}\right\rangle=\frac{1}{2}\left[\varepsilon_{y y}\left(F_{1}\right)+\varepsilon_{y y}\left(F_{2}\right)\right]$

$\left\langle\varepsilon_{z z}\right\rangle=\left[\frac{U_{z}\left(F_{1}\right)-U_{z}\left(F_{2}\right)}{t}\right]$ 
The mean strain tensors given by Equation (18) and Equation (19) are based on the strains on each face of the specimen, which can be small or large. Equation (20) gives the mean strain $\varepsilon_{z z}$ across the thickness, which is small. Then, it is consistent to use the same small strain values for the other two strain components. One would prefer to have the large strain value for $\varepsilon_{z z}$, which would lead to a better evaluation near the failure state, but this value is not easily accessible because in the localisation zone the thickness is no longer constant and uniform, but changes rapidly as one approaches failure. This makes the local strain values highly uncertain. This is the reason why we choose a small strain value from here on.

The corresponding damage (calculated from Equation (17)) is shown in Figure 16. From these results, it is clear that the volume variation and the associated damage evolution are highly nonlinear functions of time. For case $\mathrm{A}$, the damage is greater at point $\mathrm{M}$ than at point $\mathrm{O}$; for case $\mathrm{C}$, it is the opposite, although the difference is less; for case $\mathrm{B}$, it is difficult to pinpoint the location of highest damage.

Figure 17 shows the measured 'damage' distribution at the minimum section just before failure. For case $\mathrm{C}$, the slope of the damage curve is gentler than for cases $\mathrm{A}$ or $\mathrm{B}$, but the maximum damage is observed in the centre. This is probably due to the larger notch radius which leads to less strain localisation. For cases $\mathrm{A}$ and $\mathrm{B}$, the maximum damage is clearly at the edge of the specimen. The 'damage' identified by this method is about twice the critical damage identified on RB specimens. Nevertheless, let us make the following observations: First, what is called 'damage' here is, in practice, a combination of strain localisation and true damage. Second, the

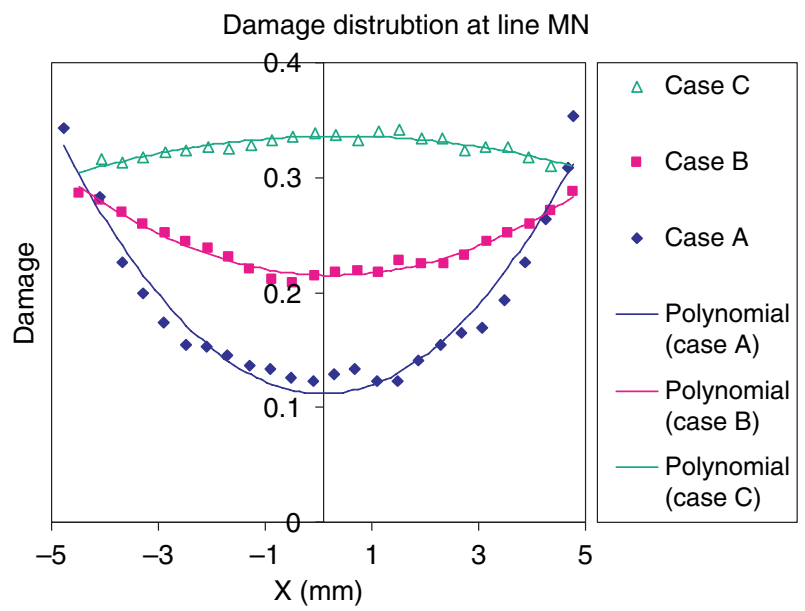

Figure 17: Damage measured along line $\mathrm{MN}$ at the time of failure

main difference between RBS tests and FNS tests resides in the size of the elementary volume being considered. In the RBS case, axial strains are assumed to be homogeneous between the sticks of the extensometer, i.e. over a $15 \mathrm{~mm}$ length. In the FNS case, strains are assumed to be homogeneous over a small pattern of $15 \times 15$ pixels, i.e. $0.75 \mathrm{~mm}^{2}$. Thus, it is clear that in the RBS case the localised postnecking phenomena are approximated only very coarsely. The stereocorrelation method used for the flat specimens is more precise.

The images at rupture recorded by the digital cameras (Figure 18C) show clearly that the initial crack occurs in the centre, then extends to the two edges. This is in good agreement with the maximum damage distribution of case $C$ (Figure 16). For cases A and $\mathrm{B}$, the initial crack could not be captured by image correlation because the specimens broke into two parts very quickly, but the cracks occurred at either point $\mathrm{N}$ or point $\mathrm{M}$.
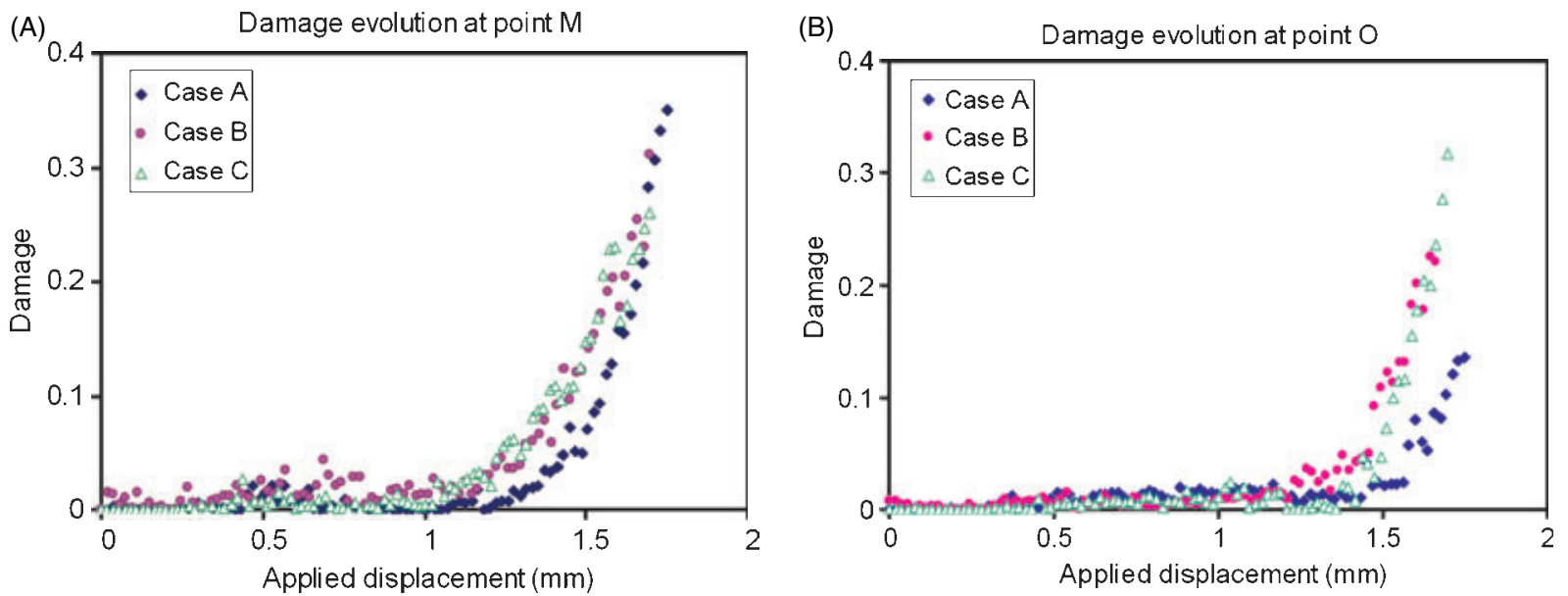

Figure 16: Measured damage at point $\mathrm{M}$ and point $\mathrm{O}$. (A) Damage at point $\mathrm{M}$; (B) damage at point $\mathrm{O}$ 
(A)

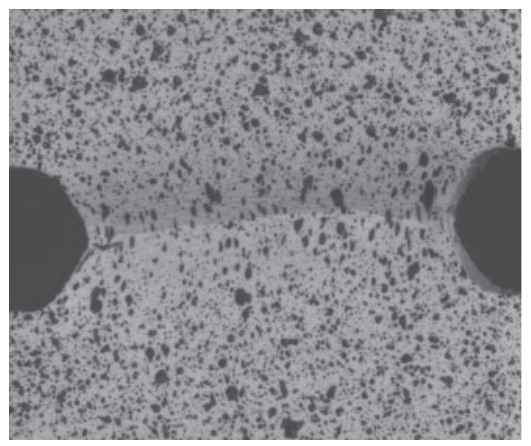

Case A
(B)

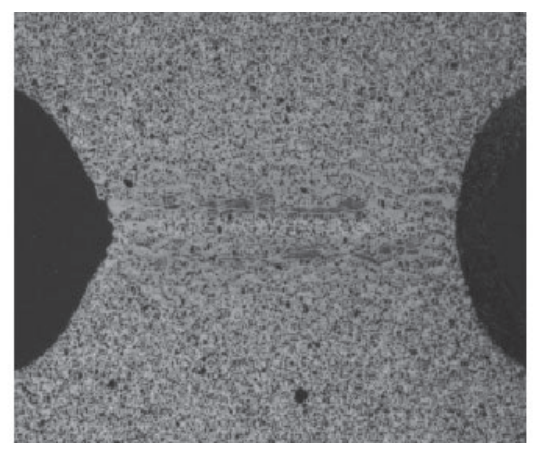

Case B
(C)

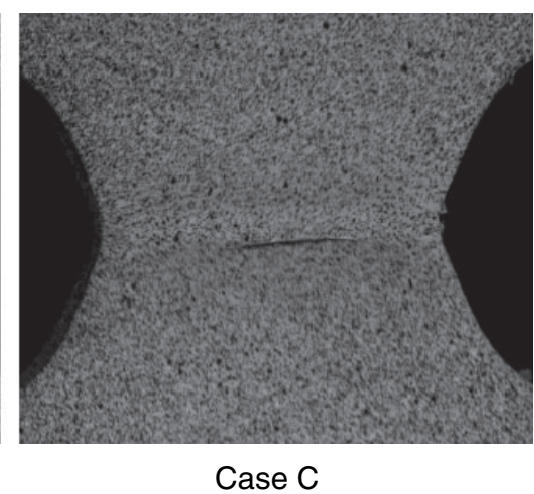

Figure 18: Macroscopic fracture observation of FNS with different notch geometries at the time of rupture. (A) Case A; (B) case B; (C) case $\mathrm{C}$

\section{Remark I}

The validity of the proposed approach for large strains could be challenged. The large-strain damage evolution is obtained directly by combining Equation (15) and Equation (17). One gets:

$D=\left(\mathrm{e}^{\operatorname{tr}(h)}-1\right)^{2 / 3}$

In our case, the strains grow exponentially close to failure and the choice of Equation (21) instead of Equation (17) changes the evaluation of damage at failure by only about $15 \%$.

\section{Remark 2}

The occurrence of a crack can also be detected in 2D situations by using DIC, or even better by using extended digital image correlation (X-DIC) [15]. This, however, was not the subject of this paper, which focuses on full 3D prediction of strain localisation prior to the occurrence of cracks.

\section{Conclusion}

The objective of the methodology presented in this paper is to measure the evolution of damage in a ductile material, from its inception to the final rupture of the specimen. The measured damage corresponds to microcracks and microvoids which grow through plastic strain, then coalesce in the final rupture stage. This was observed in the case of 15-5PH austenitic steel. The main difficulty resides in the rupture process, which is initiated in a first diffuse necking stage, then develops during a second localised necking stage. During diffuse necking, damage grows relatively homogeneously, leading to small plastic strains and a loss of stiffness. During this stage, tension tests with cyclic loading/unloading are carried out on cylindrical specimens. The measurement of the variations in the Young's modulus versus plastic strains enables one to identify damage. The main difficulty resides in the precise measurement of the strains, which relies on a relatively large measurement basis (representative elementary volume). These tests enabled us to identify a damageable model of the Lemaitre and Chaboche type, which was modified in order to take into account the nonlinearity of damage with respect to plastic strains.

Once localised necking occurs, the previous method can no longer be used because it cannot 'view' the localisation caused by the oversized measurement basis. Therefore, the proposed method consists in using a double stereocorrelation system on both faces of an FNS. Then, one can measure the strain field on each face and follow the strain evolution at each point. In addition, one gets the thickness field, which enables one, in fine, to track the volume variations and deduce the damage. The advantage of this method is that it is direct and does not require the resolution of inverse problems. Furthermore, the field measurements are local and provide a very large collection of data (several thousand) per test.

The tests carried out on $15-5 \mathrm{PH}$ showed qualitatively that the crack occurs where the damage is highest. More quantitatively, these tests provide a damage map. On this material, a critical value of the damage seems to be about 0.4 , which is twice as high as that predicted by measuring the loss of stiffness.

\section{ACKNOWLEDGEMENTS}

This research is part of the INZAT4 project dedicated to the study of the behaviour of the heat-affected zone (HAZ) during welding. INZAT4 is sponsored by EDF-SEPTEN, AREVA-NP, ESI-GROUP and EADS-CCR. 
We would like to express our gratitude to these organisations for their financial and technical support. We also thank J. Yang for his contribution to the microscopic observation. It should finally be mentioned that EADS kindly provided the $15-5 \mathrm{PH}$ stainless steel for testing purposes.

\section{REFERENCES}

1. Germain, P. and Muller, P. (1980) Introduction à la mécanique des milieux continus. Masson, France.

2. Rice, J. R. and Tracy, D. M. (1969) On ductile enlargement of voids in triaxial stress fields. J. Mech. Phys. Solids 17, 210-217.

3. Gurson, A. L. (1977) Continuum theory of ductile rupture by void nucleation and growth: Part I - yield criteria and flow rules for porous ductile media. J. Eng. Mat. Tech. 99, $2-5$.

4. Kachanov, L. M. (1986) Introduction to Continuum Damage Mechanics. Martinus, Nijhoff Publisher, Boston, MA, Dordrecht.

5. Lemaitre, J. and Chaboche, J. L. (1990) Mechanics of Solids Materials. Cambridge University Press, Cambridge.

6. Lemaitre, J. and Desmorat, R. (2005) Engineering Damage Mechanics. Springer, Berlin, Heidelberg, New York.

7. Wu, T., Coret, M. and Combescure, A. (2008) Numerical simulation of welding induced damage and residual stress of martensitic steel 15-5PH. Int. J. Solids Struct. 45, 49734989.
8. Bonora, N. (1997) A nonlinear CDM model for ductile failure. Eng. Fract. Mech. 58, 11-28.

9. Touchal, S., Morestin, F. and Brunet, M. (1997) Use of damage model and method by correlation of digital images for the necking detection. 14th Int. Conf. SMIRT, Lyon, France.

10. Brunet, M. and Morestin, F. (2001) Experimental and analytical necking studies of anisotropic sheet metals. J. Mater. Proc. Technol. 112, 214-216.

11. Choi, S. and Shah, S. P. (1997) Measurement of deformations on concrete subjected to compression using image correlation. Exp. Mech. 37, 307-313.

12. Wattrisse, B., Chrysochoos, A., Muracciole, J.-M. and Némoz-Gaillard, M. (2001) Kinematic manifestations of localisation phenomena in steels by digital image correlation. Eur. J. Mech. A/Solids 20, 189-211.

13. Helm, J. D., McNeill, S. R. and Sutton, M. A. (1996) Improved 3D image correlation for surface displacement measurement. Opt. Eng. 35, 1911-1920.

14. Cast3M, developed by the French CEA, is a computer code for structural analysis and computational fluids dynamics by the finite element method. http://www-cast3M.cea.fr [accessed on 25 December 2008].

15. Réthoré, J., Hild, F. and Roux, S. (2008) Extended digital image correlation with crack shape optimization. Int. J. Numerical Methods Eng. 73, 248-272. 